\title{
Politique publique du littoral et recherche finalisée: des pratiques et concepts en co-évolution ${ }^{\star}$
}

\author{
Nacima Baron ${ }^{*}$ \\ Géographie, Université Paris-Est Marne-la-Vallée, Laboratoire Ville Mobilité Transport, Marne-la-Vallée, France
}

Le programme « Gestion durable du littoral » (LITEAU) est un dispositif de soutien à la recherche lancé par le ministère en charge de l'environnement il y a un quart de siècle (Gautier et al., 2014). Sa marque de fabrique peut se résumer en quatre invariants qui ont structuré une demi-douzaine d'appels à projets de recherche. Il s'agissait premièrement de soutenir des projets dans lesquels les gestionnaires et les acteurs du littoral étaient réellement impliqués, deuxièmement de viser l'excellence scientifique, troisièmement de contribuer à construire en pratique une interdisciplinarité et, enfin, de développer des approches intégrées, c'est-à-dire incluant des partenaires multiniveaux dans une perspective de concertation et de coordination ${ }^{1}$.

LITEAU a permis au service de la recherche d'embrasser un sujet à caractère régalien, puisque l'espace littoral recouvre une partie du domaine public. Il est également reconnu pour la place accordée, à toutes les étapes de la production scientifique, à des partenaires parmi lesquels les services centraux et déconcentrés de l'État, ses agences (Conservatoire du Littoral, Agence des aires marines protégées...), les collectivités territoriales et les réseaux d'acteurs locaux. Ces entités sont représentées dans un comité dit d' «orientation» qui partage avec le

\footnotetext{
‡ Voir dans ce numéro le texte d'introduction de Marcel Jollivet.

* Auteur correspondant :

nacima.baron-yelles@laposte.net

${ }^{1} \mathrm{Ce}$ programme a soutenu près de quatre-vingts projets, pour une enveloppe globale d'environ 7,5 M d'euros. Le service de la recherche du ministère en charge de l'environnement et de la mer en a profité pour construire, avec l'appui de différentes communautés scientifiques dans le champ de l'océanologie, de la géologie, de l'écologie ou de la sociologie (entre autres disciplines) un cadre de recherche finalisée dans des domaines aussi variés que la gestion portuaire, la santé des mollusques, l'anticipation des risques de submersion marine ou la qualité de l'eau de baignade (Sicard, 2013).
}

comité scientifique les tâches de co-construction des appels à projets, de sélection, de suivi, de discussion et d'évaluation des projets eux-mêmes. Cette part donnée à l'expression des besoins de recherche explique en partie la très grande ouverture thématique et la diversité des types de projets qui ont réellement pu éclairer l'action publique et articuler, bien sûr à des degrés forcément divers, des enjeux de connaissance et des enjeux de gouvernance.

Cependant, pour avoir une compréhension plus fine de la conception de l'action publique et de la recherche publique qui a été mise en pratique à travers les grands axes de travail de ce programme, lui-même plusieurs fois réadapté, il faut resituer tout l'ensemble dans une trajectoire historique. C'est donc d'abord sur la façon dont la recherche a construit le littoral en tant qu'objet de recherche au gré des évolutions qui le façonnaient qu'il faut centrer notre regard pour saisir l'apport et les limites de ce programme.

\section{Cadrage scientifique: une recherche littorale en mouvement face aux dynamiques de littoralisation}

Comment construire scientifiquement le concept de littoral pour le mettre en politique en travaillant à partir d'une réalité si mouvante et instable? On saisit d'emblée combien cette contradiction est source de difficultés de compréhension et de désaccords sur les thématiques auxquelles donner la priorité dans la conduite du programme. Si le terme de littoral paraît en effet à première vue évident pour nos contemporains, les notions et les systèmes de connaissances auxquels il renvoie ont considérablement évolué.

Les choses semblaient sinon assez simples, du moins assez synchrones, au départ. La proximité entre la date de 
la loi littoral $\left(1986^{2}\right)$ et le lancement du programme (1988) laisse supposer la prise de conscience d'une nécessité d'adossement des décisions publiques à la connaissance scientifique. Et, de fait, dès ce moment, cette loi dite de protection, d'aménagement et de mise en valeur du littoral balise bien la feuille de route d'un programme scientifique censé éclairer: d'une part, au moyen de l'écologie, les conditions de mise en protection et, d'autre part, au moyen de l'océanologie (associées toutes deux aux sciences sociales), les enjeux d'occupation de l'espace et d'exploitation des ressources.

Plusieurs chocs viennent cependant rendre rapidement beaucoup plus complexes les conditions d'articulation entre, d'un côté, une recherche sur le littoral dont les concepts et les méthodes sont au départ hérités des paradigmes de l'écologie des années 1960 et 1970, et, de l'autre, la demande sociale, politique, économique de connaissances exprimée par des acteurs publics inscrits dans un contexte d'urgence croissante pour répondre à la rapidité des mutations du littoral français.

La loi littoral et la recherche sur le littoral datent globalement d'une époque dans laquelle le vocabulaire et les pratiques reflètent toute une série de partages structurants. Le terme de littoral recouvre alors trois entités étanches les unes aux autres: un pan de nature sauvage, une occupation résidentielle et des espaces productifs. Une série de règles formelles (coupure verte, logique de retrait par rapport à l'estran, construction de discontinuités latérales et longitudinales, physiques et visuelles - avec l'enjeu de co-visibilité...) sont mises en place pour tenter de protéger la première entité de la pression des deux autres en tenant compte de la variabilité de la ligne de côte (Baron, 2013). En outre, l'appréhension des enjeux de protection et de valorisation du patrimoine littoral naturel et des biens culturels sur le littoral sont pris en compte à travers des approches scientifiques disjointes (l'approche écologique d'un côté, la perspective historique et artistique de l'autre), ceci dessinant un autre grand partage. Enfin, sur le plan symbolique, le littoral fait l'objet de représentations et d'images assez manichéennes. L'urbain, l'économie, la civilisation représentent pour beaucoup d'acteurs et de chercheurs le monde du mouvement, qui progresse sur un domaine littoral vulnérable car ouvert, et plus ou moins encore perçu comme «vide» (Corbin, 1988). En conséquence, l'urgence normative consiste à tenter de sauver ce qui peut l'être dans une sorte de course contre la montre, afin de contrer la logique antagonique des faiseurs de «merlinades $»^{3}$, à travers des procédés de

\footnotetext{
${ }^{2}$ Loi n ${ }^{\circ} 86-2$ du 3 janvier 1986.

${ }^{3}$ Les « merlinades » sont les villages de vacances construits par le promoteur Merlin dans les années 1970 sur les portions de côte ouverts au tourisme de masse, et souvent dans des milieux dunaires au plus près de l'estran.
}

sanctuarisation (la préemption foncière) et des méthodes de protection plus ou moins strictes (Miossec, 1998).

Cependant, la recherche sur le littoral adopte progressivement une approche différente, allant à l'encontre de ce découpage et proposant une vision à la fois plus systémique et plus holistique. C'est moins une confrontation qu'une intégration des systèmes hommes-milieux qu'elle propose, et qu'elle analyse à travers des trajectoires de mobilité à toutes les échelles de temps et d'espace. Ce pan de la recherche est confronté, dans les années 1980, à un enjeu de mise en interaction des connaissances à propos de différents phénomènes littoraux (dynamique des processus sédimentaires, des types de populations, d'habitats...) : le défi est de pouvoir aborder les trajectoires (ou les transitions) de systèmes vivants complexes (Ministère de l'Écologie, 2014). Et cela, au moment même où le littoral français subit un basculement vers un état nouveau, marqué par une métropolisation désordonnée sous l'effet de dynamiques démographiques (mouvements migratoires vers la côte, renforcement de la densité), et de transformations économiques (recul de l'agriculture littorale traditionnelle, installations portuaires et industrielles lourdes mais ponctuelles, raz de marée touristique ou résidentiel). Ce mouvement a des causes très générales, sinon globales, et renvoie à une série de transformations économiques, sociales et culturelles de la France confrontée, avec la sortie des trente Glorieuses, à la modernité tardive (Robert et Melin, 2016).

De ce fait, la commande scientifique et politique faite à LITEAU n'est pas exempte d'une certaine ambiguité. En effet, les acteurs des politiques publiques sont tendus vers la volonté de comprendre et maîtriser les processus de littoralisation et en viennent à questionner la recherche dans des champs qui sont en partie extérieurs à ceux, habituels, de la recherche sur le littoral stricto sensu. En effet, la littoralisation ne touche pas forcément les unités spatiales directement attenantes à la mer, ce qui oblige à négocier la question de l' "épaisseur» de la «zone littorale» et à traiter des fonds d'estuaires, où justement se positionnent des métropoles puissantes. En outre, la littoralisation a autant à voir avec ce qui se produit dans l'arrière-pays ou même ailleurs (la révolution urbaine, industrielle, l'accélération des mobilités, la financiarisation de l'immobilier) qu'avec ce qui se produit sur le littoral même. À travers la demande récurrente, et relativement inaboutie, d'inscrire plus d'économie dans le programme, on repère ce décentrement progressif de la demande publique et les difficultés d'actualisation avec la - ou plutôt les - communautés scientifiques - et notamment les communautés tournées vers l'étude écologique du littoral. Ces dernières, comme on vient de le montrer, vivent de leur côté d'autres séries de ruptures épistémologiques et intègrent le système littoral dans d'autres «autres», en 
particulier sous l'angle des mutations climatiques globales et à travers le système terre-atmosphère-océan. Les chercheurs n'entendent plus tout à fait la logique des forces qui agissent et des mouvements qui en résultent de la même manière que les responsables publics. Pour nombre d'entre eux, il est difficile de rester ancré sur les grands partages, et d'opposer un système socioéconomique triomphant et avide de ressources face à un état de nature stable et un acteur public rempart. Pour ces chercheurs, tous les acteurs et les facteurs sont dynamiques et instables, déréglés et vulnérables, sur une planète où métropolisation et littoralisation vont de pair (les trois quarts de l'humanité vivent à moins de 100 kilomètres des côtes [Noin, 1999]). Prise entre ces deux historicités parallèles, la recherche sur le littoral n'est-elle pas réduite à l'impuissance du fait de la complexité de son objet?

\section{La crise permanente des politiques publiques du littoral et l'enjeu d'une recherche finalisée}

Produire une recherche finalisée destinée à éclairer les acteurs publics est aussi affaire d'intenses et permanents processus d'ajustement, et ce dès le lancement du programme. En effet, faire de la politique et faire de la science sont des démarches confrontées, dans la durée de vie de ce programme, à une crise des pratiques et des méthodes. Pour tenter de surmonter cette crise sans trop bousculer des cadres épistémologiques dont on vient d'esquisser la dualité, les responsables successifs construisent une démarche pragmatique et proposent de faire évoluer les supports d'animation, d'intervention et l'observation (Rey-Vallette et Antona, 2009). Pourquoi cette stratégie et quels en sont les résultats?

Un acteur qui aurait quitté un petit morceau de la côte française, en métropole ou en Outre-Mer, au milieu des années 1980, pour y revenir, ne serait-ce qu'au début des années 1990, pourrait difficilement appréhender l'explosion institutionnelle déclenchée par le mouvement de décentralisation et le pullulement d'acteurs et d'entités publiques et parapubliques qui ont pris part au gouvernement et à la gestion de nos côtes. Il mettrait sans doute un certain temps non seulement à cartographier ce paysage administratif, mais aussi à faire la balance entre la parole et le faire, et pour cela à pénétrer les réseaux de pouvoir et d'influence de la France maritime. La gouvernance du littoral, en effet, est un ensemble complexe d'arrangements économico-politiques particuliers qui configurent, derrière chaque dispositif public (schéma d'aménagement, programme de développement local, dynamique de branche ou de filière), des pratiques politiques et des pratiques de négociation public-privé territorialisées, reposant à chaque fois sur différents types d'accommodements avec la légalité et révélant une construction des rapports de force entre les acteurs locaux et Paris (Pennanguer, 2005). Certes, ce constat n'est pas propre à la politique du littoral, mais l'ébranlement majeur de la puissance régalienne dans le champ littoral sous l'effet de la décentralisation reconfigure aussi puissamment la demande de recherche finalisée soutenue par le Ministère. En effet, face à la montée du nombre des interlocuteurs politiques et à l'autonomisation des agendas locaux, la posture de l'Etat reste ancrée sur la défense de la loi de 1986 -tout en la faisant évoluer néanmoins (Daligaux et Rochette, 2014). L'immobilisme apparent, lié au souci de protéger le paradigme de protection lui-même, est en quelque sorte conjuré ou équilibré par l'appel aux partenariats, et par le recours au vocabulaire de la co-construction, de la participation, et de l'animation. C'est en ce sens qu'on peut analyser la montée en puissance de l'approche intégrative de la planification, de la gestion, voire de la conception des instruments d'action publique dédiés au littoral. Les bonheurs successifs de l'expression «gestion intégrée » (qu'il s'agisse de la «zone côtière » $\left(\mathrm{GIZC}^{4}\right)$, puis de la «mer et littoral» (GIML)) et la multiplication des agendas, chartes, outils d'aide à la décision ou mémoranda déclinent à l'envi cette visée et cette tension (Leclere et Bersani, 2010).

Si le nouvel enjeu de gouvernabilité du littoral ne modifie pas fondamentalement l'orientation du programme LITEAU, qui n'a jamais été un programme strictement étatique, la focalisation sur la façon de concevoir l'animation et la pédagogie participative pèse de plus en plus clairement sur les conditions de construction des projets de recherche, d'un appel à manifestation d'intérêt (AMI) à l'autre. La présence de pouvoirs locaux (les collectivités territoriales au sens large) et la montée en puissance d'agents extérieurs et de légitimités parallèles (par exemple la Fondation de France, qui porte un programme partenarial autour du littoral depuis presque dix ans) bousculent le face-à-face entre la recherche publique d'un ministère régalien et la communauté scientifique. Le contexte dans lequel les questions sont posées et les réponses doivent être adressées se modifie fondamentalement.

Sous le double effet des bouleversements territoriaux (le processus imparable de littoralisation) et institutionnels, le programme LITEAU doit donc répondre simultanément à l'expression de nouvelles injonctions venant du

\footnotetext{
${ }^{4} \mathrm{La} \mathrm{GICZ}$ est un processus dynamique qui réunit gouvernement et société, sciences et décideurs, intérêts publics et privés en vue de la protection et du développement des systèmes et ressources côtiers. Ce processus vise à optimiser les choix à long terme privilégiant les ressources et leurs usages raisonné et raisonnable (Cicin-Sain et Knecht, 1998).
} 
haut (du ministère) et du bas (sur le terrain). La recherche sur le littoral doit désormais directement interpeller la conception des politiques publiques en travaillant par exemple sur les solidarités socio-spatiales et la production des communs; elle doit participer à l'adaptation des instruments des politiques publiques aux particularités locales; elle doit trouver la manière d'inscrire les finalités environnementales dans les dynamiques territoriales, et, enfin, elle doit clairement soutenir la compétitivité des filières de production.

L'une des réponses tactiques à ces différentes prescriptions consiste à renforcer les instances d'animation à tous les niveaux (Drobenko, 2013). Au sein du programme, le chargé de mission voit sa tâche de gestionnaire de subventions évoluer progressivement vers un rôle de veille permanente, de traduction et de transfert entre les cercles scientifiques et les différents services concentrés et déconcentrés, et de structuration des procédures d'évaluation. Dans chaque projet, le responsable de la coordination des différentes équipes acquiert des compétences d'animateur. Il doit pour cela ajouter à la compétence scientifique une bonne connaissance du milieu institutionnel et socioéconomique et des comportements ou des postures de chaque groupe d'acteurs locaux, afin de les impliquer à différente étapes.

C'est réellement à ce stade que la recherche finalisée se distingue radicalement de la recherche théorique sur le littoral, à la fois par la nature des compétences qu'elle suppose et par la nature des connaissances qu'elle manipule et qu'elle produit. Dans la démarche de recueil des données ou au moment de la restitution des résultats, l'animateur scientifique devient nécessairement un passeur qui aborde le matériau de recherche de manière différente, en intégrant par exemple des connaissances locales avec des données issues de démarches scientifiques, et en rapprochant des processus de construction des savoirs très hétérogènes. Au fur et à mesure du développement du programme LITEAU, le mécanisme de co-apprentissage entre scientifiques, praticiens, acteurs locaux pour l'appropriation de la connaissance fait l'objet de dispositifs de plus en plus élaborés, et sont soumis à des dispositifs d'évaluation aussi importants que les résultats (Rocle et al., 2011). Le Ministère trouve, à travers l'intervention dans ces multiples arènes locales, non pas des résultats à transposer pour renouveler un corpus de principes, puisque ce corpus idéologique est fondamentalement stable, mais des occasions de mise en scène et de mise en parole d'une nouvelle posture que les sociologues ont fort bien analysée, et qui permet de maintenir toute une série d'équilibres entre des forces en tension (Kalaora et Konitz, 2004).

Nous en sommes arrivés à un stade dans lequel la recherche sur le littoral (et au sein de celle-ci, des formes d'une recherche produite par l'observation participative, par la co-construction des sujets et des méthodes, par le co-apprentissage des procédés de travail et par le partage des résultats) se voit dotée d'une place organique dans la production même de l'action publique. Cette forme d'intégration de la science dans l'acte de gouverner représente une étape nouvelle pour la définition de programmes de recherche relatifs au littoral. En parallèle, dès le milieu des années 2000, le Conseil national de la mer et des littoraux exprime son attachement à une recherche sur le littoral intégrée. Avec la mise en place des Conseils maritimes de façade, et la constitution du Comité interministériel de la mer (CIMer), la demande institutionnelle de recherche finalisée est encore réitérée, ce qui conduit à l'apparition d'un comité spécialisé pour la recherche marine, maritime et littorale, le COMER, qui établit dès 2014 une feuille de route dont les grandes lignes sont reprises dans le texte de la Stratégie nationale pour le littoral.

L'enjeu de la recherche sur le littoral nous paraît alors pris dans une oscillation critique. Dans un sens du balancier, la recherche finalisée en appui aux politiques publiques se centre sur un travail d'animation et de présence institutionnelle en collaborant avec les institutions scientifiques, les alliances, les institutions territoriales. De ce fait, cette recherche finalisée fait sienne la tâche qui consiste à sensibiliser, former, valider la donnée, et à la restituer à ceux qui contribuent à la constituer pour qu'ils s'approprient encore davantage les enjeux mêmes de leur action. Cette démarche induit un resserrement sur un terrain extrêmement localisé et peut conduire à des formes d' «enfermement» dans des réflexes de pensée et d'action marqués par les héritages des cadres épistémologiques et institutionnels évoqués plus haut. En particulier, l'un des réflexes les plus inconscients de cette articulation chercheurs-acteurs dans des systèmes hyper-locaux tient dans le partage d'un socle de représentations implicites comme la perception de face-à-face binaires (collectivité/État ou public/privé), et le recours quasi instinctif à une puissance étatique perçue comme nécessairement protectrice, même si on parle beaucoup d'engagement et d'empowerment. Cette construction mentale et organisationnelle de la recherche finalisée et intégrée sur le littoral confine un certain nombre de recherches dans un cadre difficilement compatible avec l'analyse des cas internationaux ou transnationaux, avec une interrogation conceptuelle ou méthodologique plus critique, et avec des bifurcations scientifiques radicales.

En ce sens, l'injonction d'une territorialisation de la recherche finalisée est contraire à une autre tendance scientifique profondément structurante. Ici, la métaphore du balancier qui oscille peut être réutilisée en évoquant le fait que les communautés de recherche sont obligées, en même temps qu'elles se territorialisent, de se dé-territorialiser pour embrasser des macro-défis 
particulièrement complexes et considérables, du fait de l'entrée du littoral dans l'ère de l'anthropocène. L'augmentation certaine, et sans doute de plus en plus catastrophique, des contraintes et des risques qui pèsent sur l'ensemble du système vivant et non vivant du littoral à toutes les échelles justifie l'urgence scientifique qui consiste à déplacer les problématiques et à diversifier les postures de recherche (Virilio, 2010). L'application du paradigme de la transition (énergétique, économique, territoriale...) au littoral oblige à construire un modèle organisationnel de production scientifique plus agile, préfigurant une autre manière d'articuler recherche et politique publique pour produire de nouvelles formes de savoirs et d'autres régimes sociaux de connaissances. Pour aborder l'état contemporain de "post-littoralisation» dont les prémisses peuvent se lire dans la ruée vers la mer, la construction d'une interdisciplinarité scientifique et technique encore plus radicale, car rejetant les découpages classiques entre sciences de la terre et du vivant, ingénierie, sciences humaines et sociales est urgente. De premiers résultats apparaissent dans le champ de la planification des façades maritimes (Cacqueray (de), 2011), car le renouveau du concept de "façade» permet de rapprocher des champs de la politique et de la science maritime d'un côté, de la politique et de la science du littoral, de l'autre, offrant donc des passerelles fertiles. Au même moment, l'espace terrestre et l'espace maritime proche mobilisent l'intérêt des développeurs (prospection énergétique, aquaculture, etc.), faisant de ce littoral non plus la base avancée d'un pays, mais une zone d'échanges entre terre et mer, arrière et avant pays, et lui redonnant un rôle d'interface plutôt que de cible (Trouillet et al., 2011). Ainsi, les travaux de planification de l'espace terre-mer menés dans la perspective de l'application de la directive européenne sur la stratégie $(\mathrm{DCSMM})^{5}$ peuvent accompagner une manière d'approcher les emprises humaines sur la terre et la mer (et donc le littoral) non mécaniquement ou thématiquement, mais en fonction de principes renouvelés, par exemple l'enjeu de l'équité spatiale et intergénérationnelle (Deldrève, 2011).

\footnotetext{
${ }^{5} \mathrm{La}$ directive 2008/56/CE du Parlement européen et du Conseil du 17 juin 2008 appelée «directive-cadre pour le milieu marin» conduit les États membres de l'Union européenne à prendre les mesures nécessaires pour réduire les impacts des activités sur ce milieu afin de réaliser ou de maintenir un bon état écologique du milieu marin au plus tard en 2020. En France, la directive a été transposée dans le code de l'environnement (articles L. 219-9 à L. 219-18 et R. 219-2 à R. 219-17) et s'applique aux zones métropolitaines sous souveraineté ou juridiction française, divisées en 4 sousrégions marines : la Manche-mer du Nord, les mers celtiques, le golfe de Gascogne, la Méditerranée occidentale.
}

Ce retour sur les relations évolutives et complexes entre les dynamiques du littoral, la recherche qui lui est dédiée et la politique publique (Bodiguel, 1997) met en valeur un programme qui a su aller bien au-delà d'un simple accompagnement des politiques publiques. Son achèvement en l'état est bienvenu au sens où il permet d'envisager une refondation (CEREMA, 20176) répondant au changement d'époque et à un changement de culture, pour répondre à une immense attente sociale d'opérationnalité, et au redoutable effort d'intelligibilité mobilisant des outils critiques notoirement plus élaborés qu'aujourd'hui. Cette démarche ne peut pas être celle, classique, de résolution de problème; elle prend acte du problème et en fait - ainsi que des conflits qu'il porte - une composante de la posture de recherche elle-même (Catanzano et Thébaud, 1995). Une telle démarche passe nécessairement par des procédures de recherche plus humbles et expérimentales, réunissant des collectifs (doctorants, chercheurs, praticiens, acteurs politiques) autour de questions émergentes et fondés sur une véritable motivation participative. De tels montages existent déjà, bien sûr, mais ils doivent être soutenus quand ils atteignent à la qualité scientifique internationale en dépassant des verrous cognitifs et en contribuant à la production de nouvelles épistémologies à la hauteur des ruptures actuelles.

\section{Références}

Baron N., 2013. Pressions anthropiques sur le littoral, in Euzen A., Eymard L., Gaill F. (Eds.), Le développement durable à découvert, Paris, CNRS Éditions, 136-137.

Bodiguel M., 1997. Le littoral entre nature et politique, Paris, L'Harmattan.

Cacqueray (de) M., 2011. La planification des espaces maritimes en France métropolitaine : un enjeu majeur pour la mise en æuvre de la Gestion Intégrée de la Mer et du Littoral, Thèse de doctorat, Brest, Université de Bretagne occidentale.

Catanzano J., Thébaud O., 1995. Le littoral, pour une régulation des conflits d'usage, Programme national d'océanologie côtière, Paris, Institut Océanographique/ IFREMER.

Cicin-Sain B., Knecht R., 1998. Integrated coastal and ocean management: concepts and practices, Washington, Island Press.

Corbin A., 2010. Le territoire du vide : L'occident et le désir du rivage Paris, Flammarion (1ère éd. 1988).

Daligaux J., Rochette J., 2014. Régulation publique de l'urbanisation : les lois de protection du littoral au défi

\footnotetext{
${ }^{6}$ Journées «Sciences et territoires » du CEREMA, organisées à Paris, les 1 et 2 février 2017 sur le thème « Mer et littoral», http://jst.cerema.fr/.
} 
de la gouvernance, in Perrin C. (Eds.), Un littoral sans nature? L'avenir de la Méditerranée face à l'urbanisation, Rome, École Française de Rome, 147-165.

Deldrève V., 2011. Préservation du littoral et inégalités écologiques, Espaces et sociétés, 1, 144-145 (173-187).

Drobenko B., 2013. Les territoires de la GIZC : rénover la vision intégrée de la mer et du littoral, VertigO, hors série 18, https://vertigo.revues.org/14304.

Gautier Q., Bélanger M., Sicard M., 2014. Apports du programme LITEAU à la gestion durable de la mer et du littoral: projets de recherche 2004-2013, Paris, MEDDE coll. « Références ».

Kalaora B., Konitz A., 2004. Le Conservatoire du littoral entre patrimonialisation et médiation, Annales de géographie, 113, 635, 87-99.

Leclere A.-S., Bersani C.D., 2010. Mer et littoral : vers une planification stratégique. Conseil général de l'Environnement et du Développement durable. Collège Paysage, Espaces protégés et Patrimoine.

Ministère de l'Écologie, 2014, État des lieux mer et littoral, Orléans, Institut français de l'environnement.

Miossec A., 1998, Les littoraux entre nature et aménagement, Paris, SEDES.

Noin D., 1999, La population des littoraux du monde, L'information géographique, 63, 2, 65-73.
Pennanguer S., 2005, Incertitude et concertation dans la gestion de la zone côtière, Thèse de doctorat, Rennes, École nationale supérieure d'agronomie.

Rey-Vallette H., Antona M., 2009, Gouvernance et gestion intégrée du littoral : l'exemple des recherches françaises, VertigO, 9, 1, https://vertigo.revues.org/8455.

Robert S., Melin, H., (Eds.) 2016, Habiter le littoral Enjeux contemporains, Aix, Presses de l'Université de Provence.

Rocle N., Lafitte A., Denis J., Henocque Y., 2011, Mesurer l'intégration dans l'action publique territoriale: l'exemple de la gestion intégrée de la mer et du littoral, $48^{\mathrm{e}}$ colloque de l'Association de Science Régionale de Langue Française (ASRDLF), 6 au 8 juillet 2011, Schoelcher, Martinique.

Sicard M., 2013, Valorisation et analyse critique de projets de recherche en appui à la gestion durable de la mer et du littoral : un bilan du programme Liteau, Mémoire de fin d'études, Rennes, Agrocampus Ouest, http://dumas.ccsd. cnrs.fr/file/index/docid/904525/filename/Sicard_Muriel_ MA moire_de_fin_d_A tudes.pdf.

Trouillet B., Guineberteau T., de Cacqueray M., Rochette J., 2011. Planning the sea : the french experience. Contribution to marine spatial planning perspectives, Marine Policy, 35, 324-334.

Virilio P., 2010, Le littoral, la dernière frontière, Revue Esprit, $12,17-24$.

Citation de l'article : Baron N., 2017. Politique publique du littoral et recherche finalisée: des pratiques et concepts en co-évolution. Nat. Sci. Soc. 25, S36-S41. 